\title{
Experimental light scattering by fluffy aggregates of magnesiosilica, ferrosilica, and alumina cosmic dust analogs
}

\author{
H. Volten ${ }^{1}$, O. Muñoz ${ }^{2}$, J. W. Hovenier ${ }^{1}$, F. J. M. Rietmeijer ${ }^{3}$, J. A. Nuth ${ }^{4}$, L. B. F. M. Waters ${ }^{1}$, and W. J. van der Zande ${ }^{5}$ \\ 1 Astronomical Institute "Anton Pannekoek", University of Amsterdam, Kruislaan 403, 1098 SJ Amsterdam, The Netherlands \\ e-mail: hovenier@science.uva.nl \\ 2 Instituto de Astrofísica de Andalucía, CSIC, Granada, Spain \\ 3 Department of Earth and Planetary Sciences, University of New Mexico, Albuquerque, New Mexico 87131, USA \\ ${ }^{4}$ Laboratory for Extraterrestrial Physics, NASA Goddard Space Flight Center, Greenbelt, Maryland 20771, USA \\ 5 Institute for Molecules and Materials, Radboud University Nijmegen, PO Box 9010, 6500 GL Nijmegen, The Netherlands
}

Received 14 November 2006 / Accepted 24 April 2007

\section{ABSTRACT}

\begin{abstract}
Context. Fluffy aggregates are generally assumed to be important constituents of circumstellar and interplanetary environments as well as to be present among the solid debris ejected from active comets.

Aims. We experimentally study light scattering properties of several fluffy aggregate samples. These cosmic dust analog aggregates are composed of coagulated magnesiosilica grains, ferrosilica grains, and alumina grains. The samples contain aggregates with different porosities. The individual grains have diameters of the order of a few tens of nanometers; the aggregates have diameters up to several micrometers.

Methods. The samples were produced in a Condensation Flow Apparatus. Their light scattering properties were measured with the Amsterdam Light Scattering Facility at a wavelength of $632.8 \mathrm{~nm}$.

Results. We measured two scattering matrix elements as functions of the scattering angle, namely $F_{11}(\theta)$ (phase function) and $-F_{12}(\theta) / F_{11}(\theta)$ (degree of linear polarization for incident unpolarized light) for seven different samples of aggregates in random orientations in an aerosol jet. The samples consisted of fluffy aggregates with cosmic dust analog compositions. We provide detailed information about their production and nature. In addition, for four of these samples we measured $F_{22}(\theta) / F_{11}(\theta)$. We covered an angle range of $5^{\circ}$ to $174^{\circ}$, in small steps of $1^{\circ}$ in the range from $5^{\circ}$ to $10^{\circ}$ and $170^{\circ}$ to $174^{\circ}$ and in steps of $5^{\circ}$ for the rest of the angle range. Conclusions. The results for the analog samples show an extremely high $-F_{12}(\theta) / F_{11}(\theta)$, with maxima between about $60 \%$ to almost $100 \%$. This Rayleigh-like behavior has been demonstrated before for fluffy aggregates and suggests that the small-sized grains in the aggregates are the main cause. Measured results for phase functions are more scarce. The phase functions we measured show shapes that are similar to those of compact micron-sized particles, suggesting that it is the overall size of the aggregates that determines their shape. The modest negative branch of $-F_{12}(\theta) / F_{11}(\theta)$ found for all seven samples seems to be mainly governed by aggregate structure. Thus, the unique combination of accurately measured phase functions and polarization functions over a fine mesh of scattering angles for cosmic dust analog aggregates enables the exploitation of the data as powerful diagnostic tools to constrain the different physical properties of dust in e.g. circumstellar clouds and in comet ejecta.
\end{abstract}

Key words. comets: general - dust, extinction - polarization - scattering

\section{Introduction}

Fluffy aggregates, i.e. loosely structured particles with high porosity, form when dust grains collide in a turbulent circumstellar dust cloud, such as the solar nebula (Cuzzi et al. 2001). Natural cosmic dust aggregates, known as interplanetary dust particles (IDPs), are routinely collected in the Earth's lower stratosphere and they include debris ejected from active comet nuclei (Zolensky et al. 1994; Rietmeijer 2002). The most likely cometary IDPs are carbon rich (Thomas et al. 1996; Flynn et al. 2003) highly fluffy aggregates (Fig. 1) but in those that survived flash heating during atmospheric entry the carbon-rich materials are often fused into contiguous patches that enclose silicate materials (Thomas et al. 1993). Thus, their pre-entry porosity was reduced by an unknown quantity.

It is not always possible to establish a direct morphological link between these surviving IDPs and aggregates not modified by flash-heating occurring in interplanetary and circumstellar environments, and cometary dust (Greenberg \& Gustafson 1981; Levasseur-Regourd et al. 1999; Petrova et al. 2000).

Recently NASA's comet sample return mission Stardust to the Jupiter Family comet 81P/Wild 2 has returned to Earth with real cometary particles. The collected dust includes the 5-10 micron terminal grains that are $\mathrm{MgFe}$-olivine, low-calcium $\mathrm{MgFe}$ pyroxenes, FeNi-sulfides, FeNi-metal and rare refractory grains (Zolensky et al. 2006; Brownlee et al. 2006). In general, particles ejected from comets or detected among circumstellar and interplanetary dust can only be studied by their interactions with light. Interpretations of the observations of these interactions will rely on a thorough knowledge of how the physical properties of the dust particles, such as size, shape, composition, and structure, influence their interactions with light. This knowledge may be obtained through experiments or model calculations. The two methods are complementary in an iterative fashion; experimental results may guide model calculations, and model calculations may aid the interpretation of experimental results. In this work 


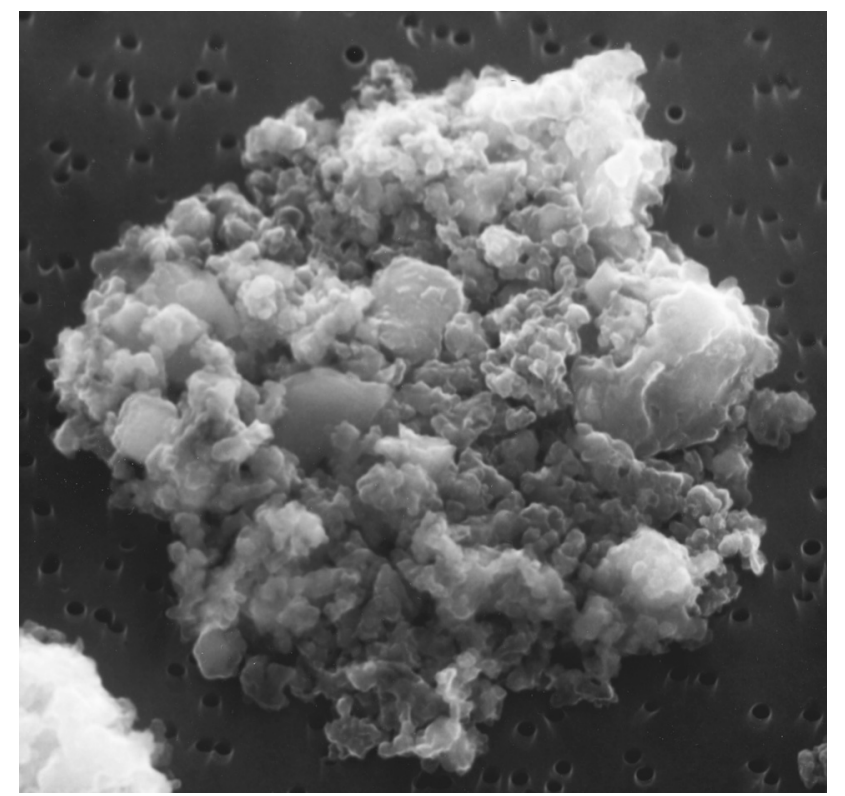

Fig. 1. Scanning electron microscope image of fluffy aggregate interplanetary dust particle W7029B13 (NASA number S-82-27575) resting on a nucleopore-filter for preliminary characterization (background). This particle is 12 micrometers in diameter. It contains platy silicate grains in a matrix of partially fused units (Rietmeijer 1998, 2002). It has an approximately chondritic bulk composition with a significant amount of light elements (e.g. carbon-rich matter). Courtesy the National Aeronautics and Space Administration.

we follow an experimental approach to study the light scattering properties of laboratory-condensed aggregate particles that will serve as analogs for cometary, interplanetary, and circumstellar dust particles.

Ideally, we would like to use ensembles of real cometary particles or IDPs in our light scattering experiments. However, at best only micrograms would be available, and we need of the order of several grams of dust to conduct the measurements in the Amsterdam Light Scattering Facility. Therefore, we take the next best option offered by cosmic dust analogs produced in the laboratory to study dust-forming processes.

Since the compositions of the individual constituents of fluffy-chondritic aggregate IDPs are still our best guide to the accreting dust in circumstellar environments, these IDPs have become the ground-truth standard for laboratory experiments. When dust coagulation is rapid, fluffy aggregates are formed with the simplest aggregates consisting of equi-granular spherical units. Such fluffy aggregates were produced in laboratory condensation experiments by coagulation of amorphous silicates for grains mostly up to $30-50 \mathrm{~nm}$ in diameter that remained after much of the condensed grains had grown via necking and fusion of the smaller grains (Rietmeijer \& Karner 1999; Rietmeijer et al. 1999a,b, 2002b). This process of grain growth is reflected by the log-normal number-size distributions of the condensed grains. The natural cosmic aggregate IDPs have a matrix of small dust grains $(10 \mathrm{~nm}$ to $\sim 1-2 \mu \mathrm{m})$ and voids with embedded larger $(\sim 5 \mu \mathrm{m})$ massive $\mathrm{Mg}$-rich silicate and $\mathrm{Fe}$, Ni-sulfide grains (Rietmeijer 1998, 2002). The grains in this matrix can have a fractal dimension (Rietmeijer 1993) and there is some evidence to suggest that these natural aggregates have fractal structure with co-increasing aggregate and constituent sizes (Rietmeijer $\&$ Nuth 2004). Aggregate IDPs have a chondritic bulk composition (i.e. solar bulk composition, cf. Anders \& Grevesse 1989) for the major elements, including $\mathrm{Si}, \mathrm{Mg}$ and $\mathrm{Fe}$ that happen to have the highest cosmic abundances among the rock-forming elements. Thus, aggregate IDPs can be almost exclusively described as mixtures of $\mathrm{Si}-, \mathrm{Mg}$ - and $\mathrm{Fe}$-oxides $\left(\mathrm{SiO}_{2}, \mathrm{MgO}, \mathrm{FeO}\right.$, $\mathrm{Fe}_{2} \mathrm{O}_{3}$ ). Laboratory condensation experiments using pre-mixed vapors of $\mathrm{Mg}-\mathrm{SiO}-\mathrm{H}_{2}-\mathrm{O}_{2}, \mathrm{Fe}-\mathrm{SiO}-\mathrm{H}_{2}-\mathrm{O}_{2}$, and $\mathrm{Mg}-\mathrm{Fe}-\mathrm{SiO}-\mathrm{H}_{2}-$ $\mathrm{O}_{2}$ bulk compositions can then be used to constrain the chemical and physical properties of circumstellar condensates and the three-dimensional aggregate structures after condensed dust coagulation. For example, the produced $\mathrm{MgSiO}$ dust compositions obtained by condensing $\mathrm{Mg}-\mathrm{SiO}-\mathrm{H}_{2}-\mathrm{O}_{2}$ vapors were used to describe processing of silicate dust grains in Herbig Ae/Be systems (Bouwman et al. 2001). In addition to such dust particles, we used alumina aggregates for our light scattering experiments, because crystalline $\mathrm{Al}_{2} \mathrm{O}_{3}$ grains could be present around oxygenrich AGB stars (Kozasa \& Sogawa 1997, 1998).

In Sect. 2 we give a brief explanation of the light scattering experimental method and we explain how the aggregates were produced and prepared. We continue in Sect. 3 with a detailed description of the cosmic analog fluffy aggregates. In Sect. 4 we present and compare the light scattering results, and in Sect. 5 we discuss a first attempt at interpreting these results. We conclude by briefly discussing the implications for astronomical observations of fluffy aggregates in space in Sect. 6.

\section{Experimental procedures}

\subsection{The Amsterdam Light Scattering Facility}

The light scattering measurements in this study were conducted with the Amsterdam Light Scattering Facility (Fig. 2). A detailed description is given by Hovenier (2000), and Hovenier et al. (2003). Here, we give a brief summary. We have used a $\mathrm{HeNe}$ laser $(632.8 \mathrm{~nm}, 5 \mathrm{~mW})$ as a light source. The laser light passed through a polarizer and an electro-optic modulator. The modulated light was subsequently scattered by an ensemble of randomly oriented particles located in a jet stream produced by an aerosol generator (see also Sect. 2.2). The scattered light passed through an optional polarization analyzer and was detected by a photomultiplier tube which moved in steps along a ring. A range in scattering angles was covered from $5^{\circ}$ (nearly forward scattering) to $174^{\circ}$ (nearly backward scattering). Another photomultiplier was used to monitor the flux of scattered light at a fixed position. We used the signal of this monitor to correct for possible fluctuations in the scattered signal. We employed polarization modulation in combination with lock-in detection to determine per measurement run three (combinations of) elements, $F_{i j}$, of the $4 \times 4$ scattering matrix, $\mathbf{F}$, of the ensemble of particles as a function of the scattering angle, $\theta$. In principle, we can measure the complete scattering matrix with the light scattering setup if enough sample is available, i.e. of the order of several tens of grams, but in the experiments we describe here the amount of material per sample was only a few grams (see Sect. 2.2). Therefore, we had to confine our measurements to a limited number of matrix elements, i.e. $F_{11}(\theta)$, and the element ratios $-F_{12}(\theta) / F_{11}(\theta)$, and $F_{22}(\theta) / F_{11}(\theta)$. Since the particles can be assumed to be randomly oriented, and time reciprocity applies (Hovenier et al. 2003), $-F_{12}(\theta) / F_{11}(\theta)$ equals the degree of linear polarization for incident unpolarized light. Further, we assumed that during the light scattering measurements the aggregates are much farther apart than their linear dimensions and, therefore, that multiple scattering by the aggregates can be neglected (Hovenier et al. 2003). The matrix elements depend on the number and physical properties of the particles, the wavelength of the incident radiation, and the direction of the scattered 


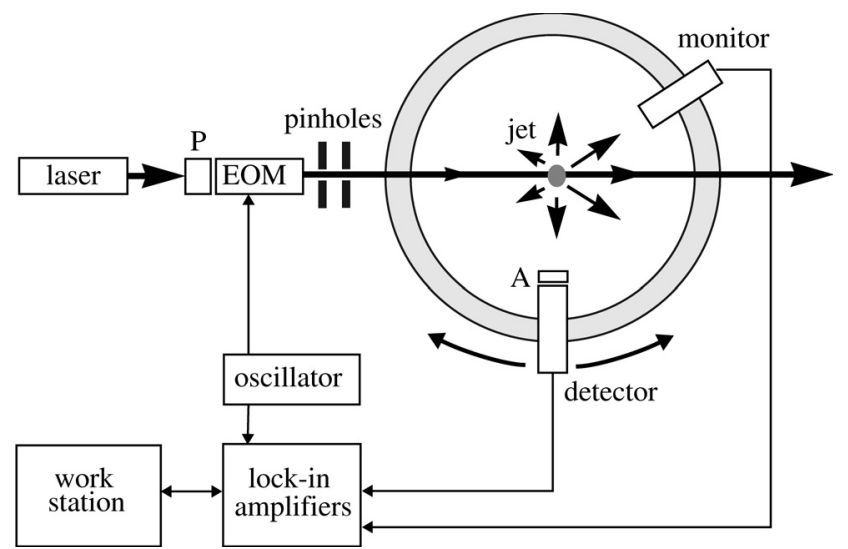

Fig. 2. Schematic picture of the light scattering setup. $\mathrm{P}=$ polarizer; $\mathrm{EOM}=$ electro-optic modulator; $\mathrm{A}=$ polarization analyzer (optional). The detector and monitor are photomultipliers. The detector can be moved in steps on a goniometer ring (shaded) with an outer diameter of about $1 \mathrm{~m}$. The aggregate particles move down in a jet stream through the nozzle of an aerosol generator in the center of the ring perpendicular to the horizontal scattering plane.

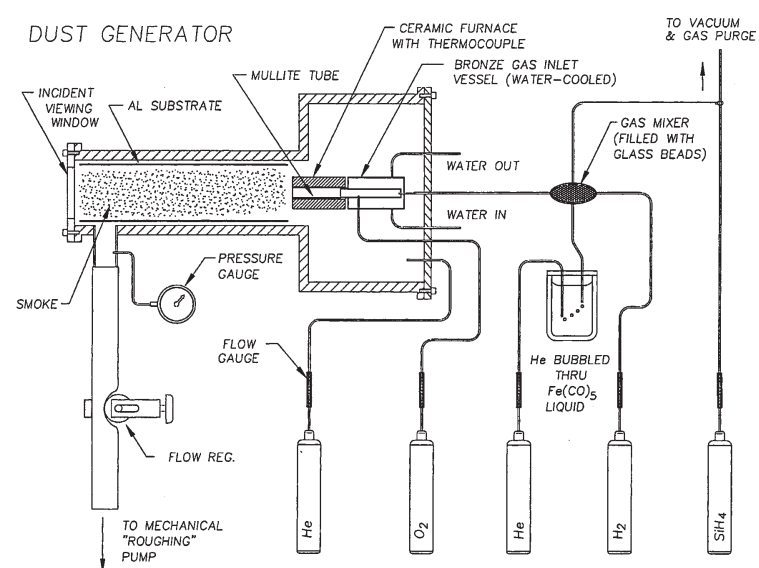

Fig. 3. Schematic diagram of the Condensation Flow Apparatus used to produce smokes of nanometer-scale grains via the combustion of hydrogen gas that contains small amounts of chemicals used to produce the desired vapor phase composition. The reaction occurs across a flame front within a resistively heated furnace at preset temperatures between 500 and $1500 \mathrm{~K}$. The rapidly quenched condensates are collected on an aluminum substrate that is placed downstream of the furnace and that is held at a nominal temperature less than $350 \mathrm{~K}$. [Reproduced from Nuth et al. (2002), with thanks to the Meteoritical Society].

light, which, for randomly oriented particles, is sufficiently described by means of the scattering angle $(\theta)$. We normalized all measured phase functions $F_{11}(\theta)$ to 1 at $30^{\circ}$. For convenience, all other matrix elements were normalized to $F_{11}(\theta)$.

\subsection{Sample preparation}

The aggregates for which we determined light scattering properties were produced in small batches in the Condensation Flow Apparatus (CFA) (Fig. 3) for experiments intended to mimic the formation of circumstellar dust (Rietmeijer et al. 1999a; Nuth et al. 2000a).

To obtain enough material (several grams) to perform our light scattering measurements, we had to combine batches of at least two condensation runs with the same bulk composition. This procedure is justifiable since the CFA condensation

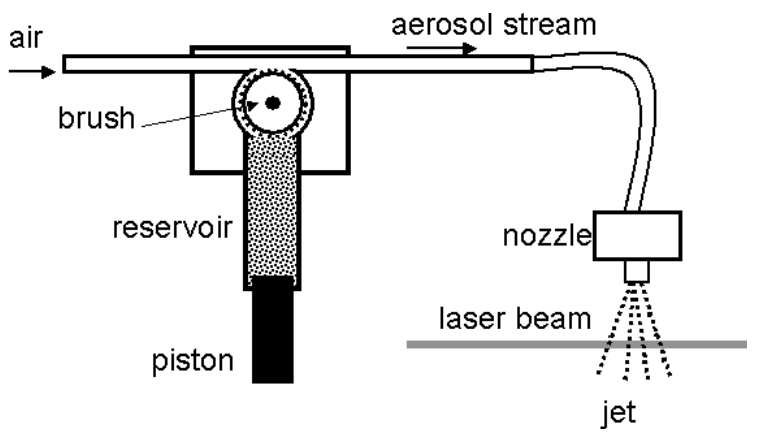

Fig. 4. Schematic diagram of the aerosol generator. The reservoir has an inner diameter of $10 \mathrm{~mm}$, and can be filled up to a height of about $70 \mathrm{~mm}$, the brush diameter is $40 \mathrm{~mm}$, the stainless steel wires of the brush are $7 \mathrm{~mm}$ long, and $0.3 \mathrm{~mm}$ in diameter. The rotation speed of the brush is $1195 \mathrm{rpm}$.

products are reproducible for condensed samples with an identical composition produced under the same experimental conditions (e.g. Rietmeijer \& Karner 1999; Rietmeijer et al. 1999b, 2002b). The individual experimental batches were placed in a container and stirred together with a spatula to obtain a homogeneous mixture. A complicating factor in handling the samples is that they consisted of highly electrostatic fluffy aggregates of nanograins. Some condensed material when removed from the collector plates placed inside the Condensation Flow Apparatus consisted of very large, up to millimeter-size agglomerates that even upon the slightest touch would disintegrate.

For previous light scattering measurements we used samples of compact particles with sizes of the order of micrometers of either natural origin, e.g. volcanic dust particles (Muñoz et al. 2004), and desert dust particles (Volten et al. 2001), or samples obtained after physical comminution of larger rocks, e.g. forsterite (Volten et al. 2005, 2006a,b). However, the fluffy aggregate samples are of a different nature; they are ultrafinegrained coherent powders and required a new approach for preparing the samples for the aerosol generator (Fig. 4). Our novel method involved compacting each sample carefully but tightly inside the sample reservoir, which in case of the coarser compact particles would have caused damage to the aerosol generator. This sample preparation procedure was a prerequisite to ensure a stable aerosol jet. The aggregate sample thus prepared inside the reservoir was then pushed slowly $(\sim 25 \mathrm{~mm} / \mathrm{h})$, in a controlled manner, against a fast rotating (1195 rpm) fine-wire steel brush and subsequently blown off of the brush into a jet that flows through the light scattering center of the experimental setup. SEM images of aggregates collected from the aerosol jet show that the original massive millimeter-sized agglomerations of aggregates had been destroyed during this procedure. The SEM images (Fig. 5) also show evidence that the original aggregate structure had not been destroyed nor had caused changes in the number-size distribution of the condensed nanograins.

In total we produced seven aggregate samples with compositions as listed below.

1. dark-brown magnesiosilica indicative of a high amount of amorphous $\mathrm{MgSiO}$ plus crystalline $\mathrm{MgO}$ grains; $\mathrm{Mg} / \mathrm{Si}=0.5$;

2. same as 1 , but with $\mathrm{Mg} / \mathrm{Si}=1.4$;

3. light-brown magnesiosilica indicative of amorphous $\mathrm{MgSiO}$ with a high amount of silica grains; $\mathrm{Mg} / \mathrm{Si}=1.3$;

4. same as 3, but no hydrogen in the condensing vapor;

5. black ferrosilica; 


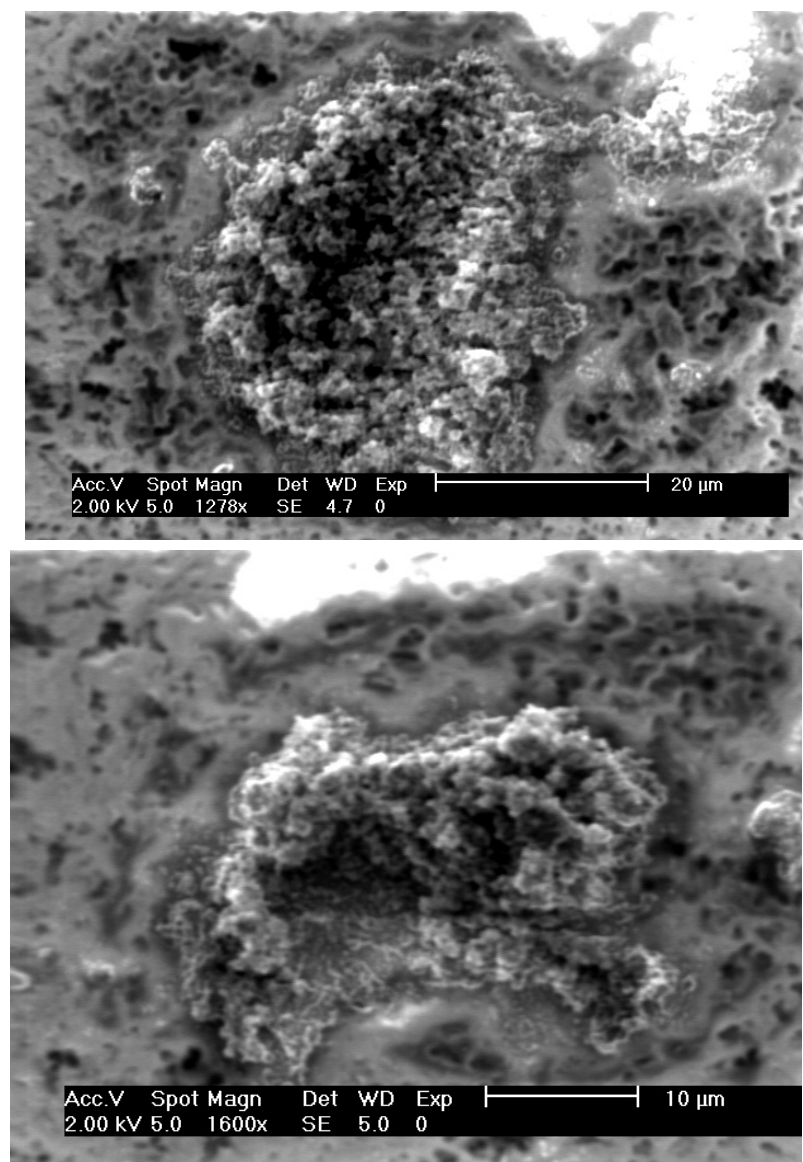

Fig. 5. SEM images of large and several small magnesiosilica aggregate particles (sample 1, top; and sample 3, bottom) placed on a carbon filter. The linear features in both images are an instrumental artifact due to sample charging caused by the low voltage $(2 \mathrm{keV})$ incident electron beam on samples that were not provided with a conductive coating.

6. black ferrosilica-magnesiosilica mixture, condensed from a vapor mixture containing $\mathrm{Mg}$ and $\mathrm{Fe}$;

7. white alumina.

In this table, $\mathrm{Mg} / \mathrm{Si}$ refers to this ratio of the $\mathrm{Mg}-\mathrm{SiO}-\mathrm{H}_{2}-\mathrm{O}_{2}$ vapor. While this ratio will affect the relative amounts of $\mathrm{MgO}$ and $\mathrm{SiO}_{2}$ condensates, its main impact will be on the relative amounts of the mixed $\mathrm{MgSiO}$ compositional groups (Rietmeijer $\&$ Nuth 2004) of the smallest grains in the samples. Their relative proportions do not induce textural changes in the bulk sample and we exclude this chemical effect from our considerations. All but sample 4 were condensed in $\mathrm{H}_{2}$-containing vapors but with slightly variable $\mathrm{O}_{2} / \mathrm{H}_{2}$ ratio. The effect, if any, on the overall bulk texture is unknown.

In this paper silica, which is the dioxide of silicon, is used for amorphous $\mathrm{SiO}_{2}$ condensates but, when crystalline, we use the appropriate polymorph designation; in this study that is tridymite. Silicon dioxide in combination with other metaloxides, e.g. $\mathrm{MgO}$ or $\mathrm{FeO}$, will form more complex compounds. They can be either amorphous or crystalline solids. In the latter, $\mathrm{SiO}_{4}$ tetrahedra become building blocks of the crystal lattice of silicate minerals, such as forsterite and enstatite. The term "amorphous silicate(s)" is strictly speaking incorrect but is used in astronomy for a compound in which the 10 and 20-micron infrared (IR) features are due to the lack of a crystal lattice. In this paper, we use this term for any compound that contains silicon dioxide in combination with other oxides but that is
Table 1. Real parts of the refractive index.

\begin{tabular}{lll}
\hline \hline mineral & $n$ & comments \\
\hline $\mathrm{SiO}_{2}$ glass & 1.46 & appropriate for our aggregates \\
$\mathrm{MgSiO}_{3}$ & 1.65 & crystalline enstatite; upper limit \\
$\mathrm{Mg}_{2} \mathrm{SiO}_{4}$ & 1.66 & crystalline forsterite; upper limit \\
$\mathrm{MgO}$ & 1.74 & crystalline periclase; upper limit \\
$\mathrm{FeSiO}_{3}$ & 1.77 & crystalline pyroxene; upper limit \\
$\mathrm{Fe}_{2} \mathrm{SiO}_{4}$ & 1.87 & crystalline fayalite; upper limit \\
$\mathrm{Fe}_{2} \mathrm{O}_{3}$ & 3.0 & crystalline hematite; upper limit \\
$\mathrm{Al}_{2} \mathrm{O}_{3}$ & 1.66 & glass phase alumina; upper limit \\
\hline
\end{tabular}

crystallograhically amorphous. For the condensed smokes used in the present study, this particular issue of nomenclature is described in two separate IR (Hallenbeck et al. 1998, 2000) and transmission electron microscope (Rietmeijer et al. 2002a) studies of the post-condensation thermal annealing of amorphous $\mathrm{MgSiO}$ samples.

\subsubsection{Refractive indices}

With the exception of $\mathrm{SiO}_{2}$, quantitative standard values for the refractive indices of the largely amorphous grains and aggregates are not available. The degree of solid disorder in our amorphous condensed aggregates will be considerably larger than in the amorphous quenched-liquid glasses in the excellent Jena database of refractive indices (Henning et al. 1999). Thus, we anticipate that the values in this database will be lower than the refractive indices of our vapor-condensed samples. As far as the imaginary parts of the refractive index are concerned, these values may vary orders of magnitude even within one mineral (Egan \& Hilgeman 1979). For the real parts of the refractive index $n$ useful estimates can be extracted from Deer et al. (1974) and Weast et al. (1998) for indices of refraction that were measured in the Na-line at room temperature. These values are reproduced in Table 1.

For amorphous or glassy materials the real parts of the refractive index are usually smaller than for crystalline materials. Therefore, the values for the crystalline silicates (forsterite; enstatite; pyroxene), and also the glass phase alumina in Table 1 will serve as an upper limit for, respectively, the amorphous $\mathrm{MgSiO}, \mathrm{FeSiO}$, and $\mathrm{AlO}$ materials.

\section{Sample descriptions}

\subsection{Amorphous silicate aggregates}

The magnesiosilica samples $1-4$, the ferrosilica sample 5 and the ferrosilica-magnesiosilica sample 6 were prepared by condensing high $\mathrm{SiO} / \mathrm{metal}$ vapors in the Condensation Flow Apparatus (Nuth et al. 2000a,c). SiO molecules were the dominant gas phase species in samples 1-6 (Nuth \& Donn 1984) that will react with "MgO" or "FeO" vapor molecules, or both (Nuth \& Donn 1983) but with as yet unknown metal/oxygen vapor ratios. During condensation an initial mixture of condensed $\mathrm{Si}_{2} \mathrm{O}_{3}$ and $\mathrm{SiO}_{2}$ molecules (Nuth \& Donn 1984) may continue to react with other condensing species. Ultimately silica is present as $\mathrm{SiO}_{2}$ condensates. The compositions and crystallographic properties of the condensates show well-defined systematic trends (Rietmeijer et al. 1999a; Nuth et al. 2002). A typical condensate will contain two types of grains, viz. (1) (non-mixed) simple metal-oxide grains $\left(\mathrm{SiO}_{2}, \mathrm{MgO}, \mathrm{Fe}_{2} \mathrm{O}_{3}\right)$, and (2) mixed grains with magnesiosilica $(\mathrm{MgSiO})$ or ferrosilica $(\mathrm{FeSiO})$ compositions, or combinations thereof. The invariably predominating 
mixed grains are arranged in chains, i.e. the common and typical "necklaces" that have been reported for vapor condensation experiments for many years since scanning or transmission electron microscopes were used for their characterization (e.g. Krikorian \& Sneed 1979, among many others). In our samples, the chains form interconnected, tangled 3D-networks ranging from (very) open structures to dense structures (see Fig. 6). Mixed grains ranging from $\sim 2 \mathrm{~nm}$ up to $\sim 100 \mathrm{~nm}$ show lognormal number-size distributions. In most samples the mixed grains define two log-normal number-size distributions with a discontinuity between diameters of 30 to $50 \mathrm{~nm}$. The samples prepared for Transmission Electron Microscope (TEM) studies of the condensed aggregates consisted of particles that rarely contain mixed grains and silica larger than about $100 \mathrm{~nm}$. Mixed grains are typically amorphous but rare forsterite $\left(\mathrm{Mg}_{2} \mathrm{SiO}_{4}\right)$ nanocrystals can be present. The ferrosilica aggregate sample 5 has similar chemical and crystallographic properties of its individual grains (Rietmeijer \& Nuth 1991), but this sample has a more open, very loose structure since interconnected, tangled 3D-networks have developed to a much lesser degree than in a magnesiosilica aggregate (see Sect. 3.3). Although it was formed from a vapor mixture containing both iron and magnesium, sample 6 does not contain mixed ferromagnesiosilica grains (Rietmeijer et al. 1999a; Rietmeijer 2002). Instead, this sample is an intimate mixture of condensed magnesiosilica and ferrosilica domains.

Each of the samples 1-6 contains simple metal-oxide grains in addition to mixed, amorphous silicate grains. In these samples amorphous silica grains occur in chains, generally forming open structured domains, or they occur in dense agglomerations mostly $\sim 150 \mathrm{~nm}$ in size. In addition, larger massive, texturally homogenous, (sub)spherical silica blobs can be found interspersed with mixed silica aggregate domains. Pure $\mathrm{MgO}$ condensates form both single-crystal periclase grains and massive, rounded, amorphous $\mathrm{MgO}$ grains.

The color difference between the dark-brown magnesiosilica samples 1 and 2 and the light-brown magnesiosilica samples 3 and 4 reflect variations in the relative amounts of condensed $\mathrm{SiO}_{2}$ and amorphous, non-stoichiometric $\mathrm{MgSiO}$ grains, and, to a lesser degree, also variable contents of periclase grains. (Nonstoichiometric means the elements do not occur in the exact proportions of the well-ordered mineral, e.g. $\mathrm{Mg}_{2} \mathrm{SiO}_{4}$ (forsterite) and $\mathrm{MgSiO}_{3}$ (enstatite).) In general, a light color indicates a high number of pure silica condensates (about 25\%) relative to $\mathrm{MgSiO}$ grains, whereas a dark color is indicative for an overwhelmingly high abundance of condensed $\mathrm{MgSiO}$ plus $\mathrm{MgO}$ grains $(<5 \%)$.

The iron-oxide in samples 5 and 6 also causes a dark color. The iron oxide forms (sub)spherical, single-crystal magnetite and maghémite; both $\mathrm{Fe}_{2} \mathrm{O}_{3}$ polymorphs (Rietmeijer et al. 2006). The invariably crystalline $\mathrm{MgO}$ and $\mathrm{Fe}_{2} \mathrm{O}_{3}$ condensates occur as individual grains or as compact clusters of several $\mathrm{MgO}$ or $\mathrm{Fe}_{2} \mathrm{O}_{3}$ grains interspersed throughout an aggregate sample.

\subsection{Alumina aggregates}

To produce the alumina aggregate sample 7 , we condensed an $\mathrm{Al}-\mathrm{H}_{2}-\mathrm{O}_{2}$ vapor in the same CFA experimental set-up using $\mathrm{Al}\left(\mathrm{CH}_{3}\right)_{3}$ as the source for $\mathrm{AlO}$ vapor molecules. The experiment yielded widely scattered, isolated grains and grain clusters indicating an extremely loosely structured aggregate (Fig. 6c). At the macroscopic scale this condensed sample consists of very loose, fragile aggregates of individual grains that are typically $<75 \mathrm{~nm}$ in diameter with a modal diameter around
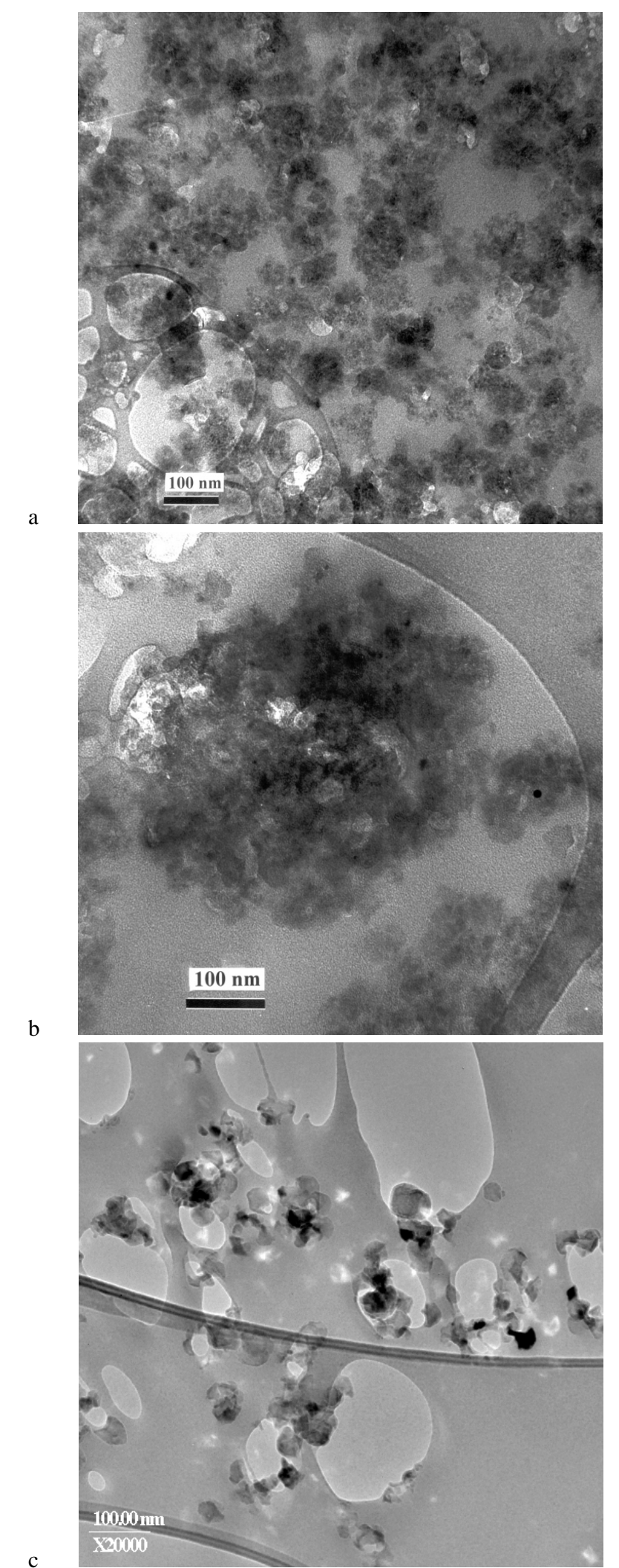

Fig. 6. a), b) Two TEM images of ultrathin $(90 \mathrm{~nm})$ sections sliced through a fluffy $\mathrm{MgSiO}$ particle consisting of condensed magnesiosilica grains organized in the form of small aggregates. c) A TEM image of an ultrathin $(70 \mathrm{~nm})$ section sliced through an alumina aggregate showing the typical very open structure indicating a highly fluffy aggregate sample. The dark-grey to white features are the holey-carbon thin film that supports the ultrathin sections of epoxy with embedded aggregate material during TEM analyses. 
$50 \mathrm{~nm}$. When exposed to the $200 \mathrm{keV}$ incident electron beam in the high-resolution transmission electron microscope the amorphous Al-O grains rapidly developed a vesicular, "boiling"-like, structure. Compositionally the Al-O grains were stoichiometric $\mathrm{Al}_{2} \mathrm{O}_{3}$ but statistically significant deviations of oxygen deficiency occur that tend to be correlated with a developing vesicular structure. Al-oxide grains condensed in $\mathrm{Fe}-\mathrm{Al}-\mathrm{SiO}-\mathrm{H}_{2}-\mathrm{O}_{2}$ vapors showed similar loose structures (Rietmeijer et al. 2006). Gel-produced amorphous $\mathrm{Al}_{2} \mathrm{O}_{3}$ showed a loose structure but, in this case, the structure was probably filled with liquid water (Begemann et al. 1997). It appears that the loose structure for amorphous alumina does not depend on the production technique.

\subsection{Aggregate fluffiness}

Macroscopically, all our samples consist of fluffy aggregates of the components discussed above that are mixed in relative proportions as a function of vapor phase bulk composition. The individual components themselves, in particular the amorphous mixed silicate domains and amorphous silica domains, form aggregates of very different porosities (see above). The magnesiosilica samples are produced from vapors that are rich in $\mathrm{SiO}$ and $\mathrm{Si}_{2} \mathrm{O}_{3}$ vapor molecules to facilitate the condensation of mixed amorphous grains with $\mathrm{MgSiO}$ compositions. Chemical diffusion will be simple and neck formation is probably rapid. Although we do not expect all magnesiosilica samples 1-4 to have exactly the same fluffiness, we will use their estimated fluffiness as a qualitative reference at this time. In the condensing $\mathrm{Fe}-\mathrm{SiO}-\mathrm{H}_{2}-\mathrm{O}_{2}$ vapors, also rich in $\mathrm{SiO}$ and $\mathrm{Si}_{2} \mathrm{O}_{3}$ vapor molecules, iron oxidation results in variable $\mathrm{Fe}^{2+} / \mathrm{Fe}^{3+}$ ratios of the mixed ferrosilica grains (Rietmeijer et al. 2006). We conjecture that the variable $\mathrm{Fe}^{2+} / \mathrm{Fe}^{3+}$ ratios contributed to the lining-up of the nanograins in isolated narrow strands in favor of forming denser structures. This effect could explain the observed very open structure of the aggregates (Rietmeijer \& Nuth 1991; Rietmeijer et al. 1999b) and the more open structures of ferrosilica aggregates in samples 5 and 6 compared to magnesiosilica aggregates in samples $1-4$.

The absence of $\mathrm{SiO}$ and $\mathrm{Si}_{2} \mathrm{O}_{3}$ vapor molecules during condensation of the alumina aggregates likely retarded the onset of AlO-condensate formation. The resulting vapor supersaturation resulted in extremely fluffy aggregates of amorphous $\mathrm{Al}_{2} \mathrm{O}_{3}$ grains (Fig. 6c). At the macroscopic scale sample 7 consists entirely of very loose and fragile aggregates. Its fluffiness is even greater than that of ferrosilica sample 5.

\subsection{Grain and aggregate sizes}

Unfortunately laser diffraction methods to measure size distributions, which are very useful for small compact particles (Konert \& Vandenberghe 1997), are extremely difficult to use for the fluffy particles studied here, for two main reasons. First, the fluffy aggregates are highly hygroscopic and therefore cannot be dispersed in water as is usually needed in laser diffraction instruments. Second, these fluffy aggregate particles are fragile. The condensing grains are continuously depositing onto collector surfaces that are placed inside the CFA condensation chamber (see Fig. 3). When removed from the collector, these deposits are typically highly electrostatically charged agglomerations of aggregates of several millimeters and larger in size. As mentioned in Sect. 2.2, these agglomerations are broken up inside the aerosol generator into numerous fluffy aggregates prior to the actual light scattering measurements.

Although it is not possible to obtain measured number-size distributions in the same way as for compact particles (Volten et al. 2005, 2006b), we have obtained information about the dimensions of grains and aggregates by studying TEM images. We have to keep in mind that for these fluffy particles it is often difficult to make a clear distinction between what is a grain and what an aggregate. For example, whether several small spheres fused together form a grain or a small aggregate is hard to tell. Therefore, what we call grain sizes and aggregate sizes may be overlapping quantities, because many intermediate forms exist in these samples.

From TEM analyses we know that the mixed amorphous silicate and amorphous silica grains, forming chains and aggregates with widely different porosities, range in diameter from $\sim 2 \mathrm{~nm}$ to $\sim 100 \mathrm{~nm}$; the modal diameter is about $50 \mathrm{~nm}$ (see also Fig. 6). Dense, low-porosity, amorphous silica aggregates range from $30-50 \mathrm{~nm}$ (in smoke-like chains) up to $150-400 \mathrm{~nm}$. The smallest recognizable grains are invariably spherical and this is still the case for most grains up to about $50 \mathrm{~nm}$. Larger dense aggregates tend to show irregular circumferences on TEM-images when they consist of incompletely fused small grains. It is particularly the case for mixed $\mathrm{MgSiO}$ dense aggregates that can be as large as $\sim 650 \mathrm{~nm}$. In the ferrosilica sample 5 such dense aggregates are typically $<400 \mathrm{~nm}$. Dense aggregates may contain up to several hundreds of grains. The pure $\mathrm{MgO}$ grains are $40-170 \mathrm{~nm}$ in diameter (average $\sim 120 \mathrm{~nm}$ ); the $\mathrm{Fe}_{2} \mathrm{O}_{3}$ grains are typically $<80 \mathrm{~nm}$ (average $\sim 45 \mathrm{~nm}$ ). The micrometer-sized aggregates produced inside the aerosol generator (Fig. 4) are mixtures of such grains. For completeness we mention that the alumina sample 7 only has smooth amorphous $\mathrm{Al}_{2} \mathrm{O}_{3}$ grains, smaller than about $75 \mathrm{~nm}$ in diameter.

\section{Light scattering results}

Experimental results for the magnesiosilica samples 1-4 in the scattering angle range $5^{\circ}$ to $174^{\circ}$ are shown in Fig. 7 and Table 2 . Clearly, the measured phase functions of these 4 samples are similar, i.e. smooth and strongly peaked in the forward directions. For these normalized phase functions we distinguish two groups of behavior, i.e. the two light-brown samples (samples 3 and 4) lie below the two dark-brown samples (samples 1 and 2) for large scattering angles. This grouping is also apparent if we take into account the steepness, expressed in Table 2 as the ratio of $F_{11}\left(10^{\circ}\right)$ and $F_{11}\left(90^{\circ}\right)$. The two light-brown samples 3 and 4 have a higher steepness than the dark-brown samples 1 and 2 . Grouping according to color is less obvious for the degree of linear polarization for incident unpolarized light $-F_{12}(\theta) / F_{11}(\theta)$, although the light-brown magnesiosilica samples 3 and 4 show a higher maximum than the dark-brown samples 1 and 2 . The degree of linear polarization for unpolarized incident light shows a bell shaped curve with a high maximum around $90^{\circ}$ and a small negative branch of around 1 to $2 \%$ close to the backward direction for all four magnesiosilica samples. The maxima for $-F_{12}(\theta) / F_{11}(\theta)$ range from $57 \%$ to $86 \%$ (see Table 2). For these four magnesiosilica samples we have also measured $F_{22}(\theta) / F_{11}(\theta)$ ratios. For lack of material only one measurement was performed per sample. Therefore, no error bars could be determined for the $F_{22}(\theta) / F_{11}(\theta)$ ratios. We observe the same grouping as for $F_{11}(\theta)$, i.e. at large scattering angles the values of $F_{22}(\theta) / F_{11}(\theta)$ of the light-brown samples 3 and 4 are above those of the dark-brown samples 1 and 2 in Fig. 7 (see also Table 2). 

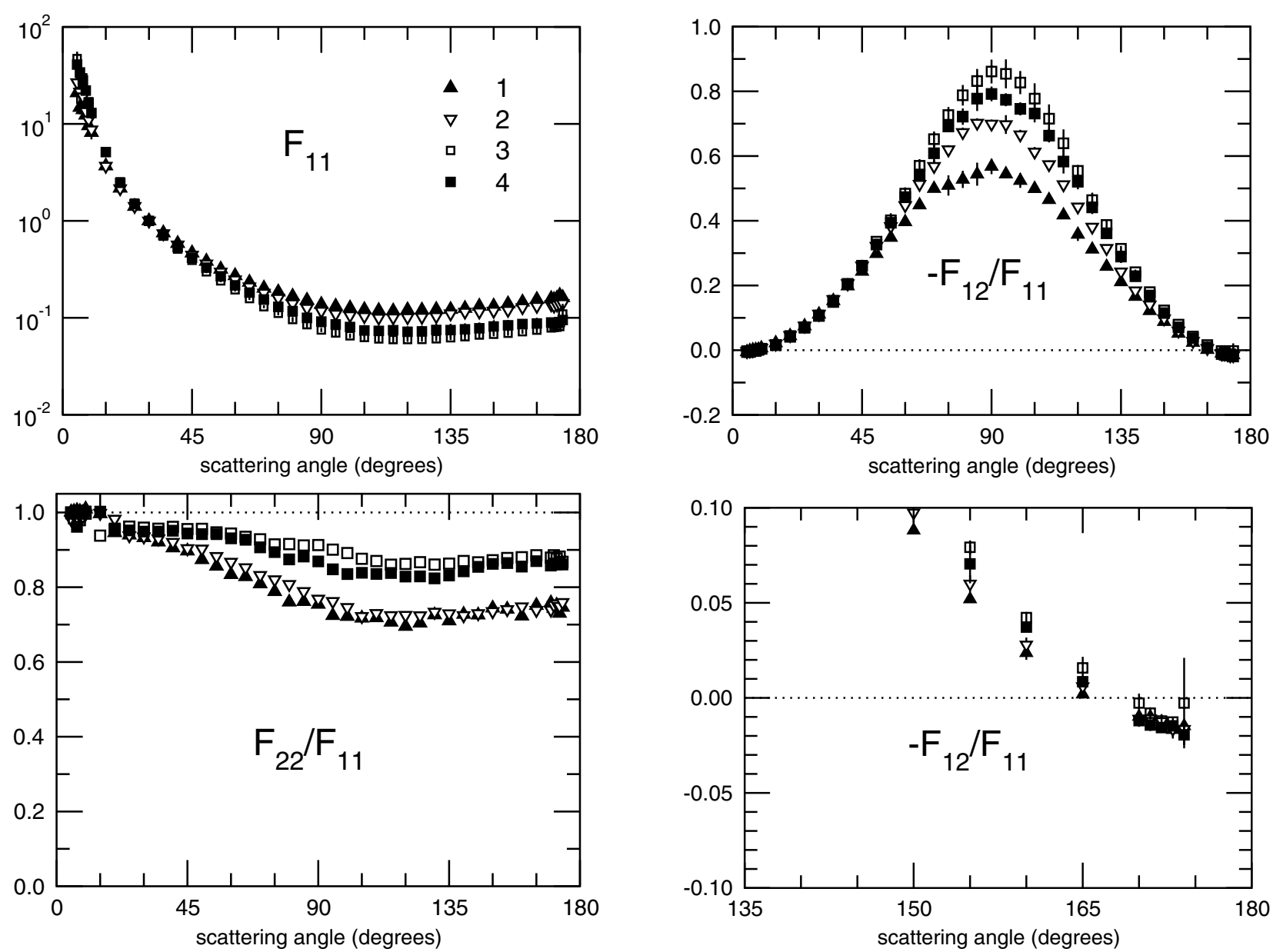

Fig. 7. Measured angular distributions of the scattering matrix elements $F_{11}(\theta),-F_{12}(\theta) / F_{11}(\theta)$ and $F_{22}(\theta) / F_{11}(\theta)$ at $632.8 \mathrm{~nm}$ for magnesiosilica samples 1-4. Triangles up (sample 1) and down (sample 2) are for two dark-brown samples, open (sample 3) and solid (sample 4) squares are for two light-brown samples. For $F_{11}(\theta)$ and $-F_{12}(\theta) / F_{11}(\theta)$, if no error bars are visible, errors are smaller than the symbols. For $F_{22}(\theta) / F_{11}(\theta)$ for lack of material only one measurement was performed per sample. Therefore, no error bars could be determined. In the bottom right panel an enlargement of the measured $-F_{12}(\theta) / F_{11}(\theta)$ at large scattering angles is shown. The data can be found in numerical form in "The Amsterdam Light Scattering Database" (http://www. astro.uva.nl/scatter).

Table 2. Matrix element ratios.

\begin{tabular}{llll}
\hline \hline Sample & $\frac{F_{11}\left(10^{\circ}\right)}{F_{11}\left(90^{\circ}\right)}$ & $-\frac{F_{12}\left(90^{\circ}\right)}{F_{11}\left(90^{\circ}\right)}$ & $\frac{F_{22}\left(174^{\circ}\right)}{F_{11}\left(174^{\circ}\right)}$ \\
\hline 1 & $59 \pm 4$ & $0.57 \pm 0.02$ & 0.75 \\
2 & $74 \pm 16$ & $0.70 \pm 0.01$ & 0.76 \\
3 & $175 \pm 16$ & $0.86 \pm 0.04$ & 0.87 \\
4 & $142 \pm 26$ & $0.79 \pm 0.02$ & 0.86 \\
5 & $236 \pm 57$ & $0.66 \pm 0.03$ & \\
6 & $233 \pm 5$ & $0.71 \pm 0.001$ & \\
7 & $718 \pm 34$ & $0.98 \pm 0.02$ & \\
\hline
\end{tabular}

In Fig. 8 light scattering results are shown for the black ferrosilica sample 5, the black ferrosilica-magnesiosilica mixture sample 6 , and the white alumina sample 7 . As a reference, the results from Fig. 7 are indicated in grey. The results for $F_{11}(\theta)$ of the two samples 5 and 6 almost coincide, and practically overlap the light-brown magnesiosilica results, although their steepness as given in Table 2 is significantly higher. For $-F_{12}(\theta) / F_{11}(\theta)$ the results of samples 5 and 6 are close to the dark-brown magnesiosilica results. The alumina sample 7 shows a quite different behavior. The $F_{11}(\theta)$ curve of this sample stands out for its extreme steepness (see Table 2) and $-F_{12}(\theta) / F_{11}(\theta)$ shows an extremely high maximum of almost $100 \%$.

All seven samples have a small negative branch of about $1-2 \%$ at large scattering angles (Fig. 8).

\section{Discussion}

We start by summarizing in Table 3 the composition, real part of the refractive index (n) and color, fluffiness, and the grain and aggregate sizes, which are considered to be the main parameter candidates determining the light scattering behavior of the seven analog samples.

Before we discuss the differences in scattering behavior between the seven dust analog samples, we will first look at their general behavior. All of the seven phase functions (Fig. 8) are strongly peaked towards small scattering angles and rather flat at large scattering angles. This behavior resembles that observed for micron-sized compact irregular particles containing large fractions of silicate (Volten et al. 2005, 2006b). In contrast, the $-F_{12}(\theta) / F_{11}(\theta)$ ratio shows much higher maxima, and for the condensed $\mathrm{MgSiO}$ samples much higher minima of $F_{22}(\theta) / F_{11}(\theta)$, than seen for e.g. the compact irregular olivine particles (Muñoz et al. 2000; Volten et al. 2005, 2006b). Similar 

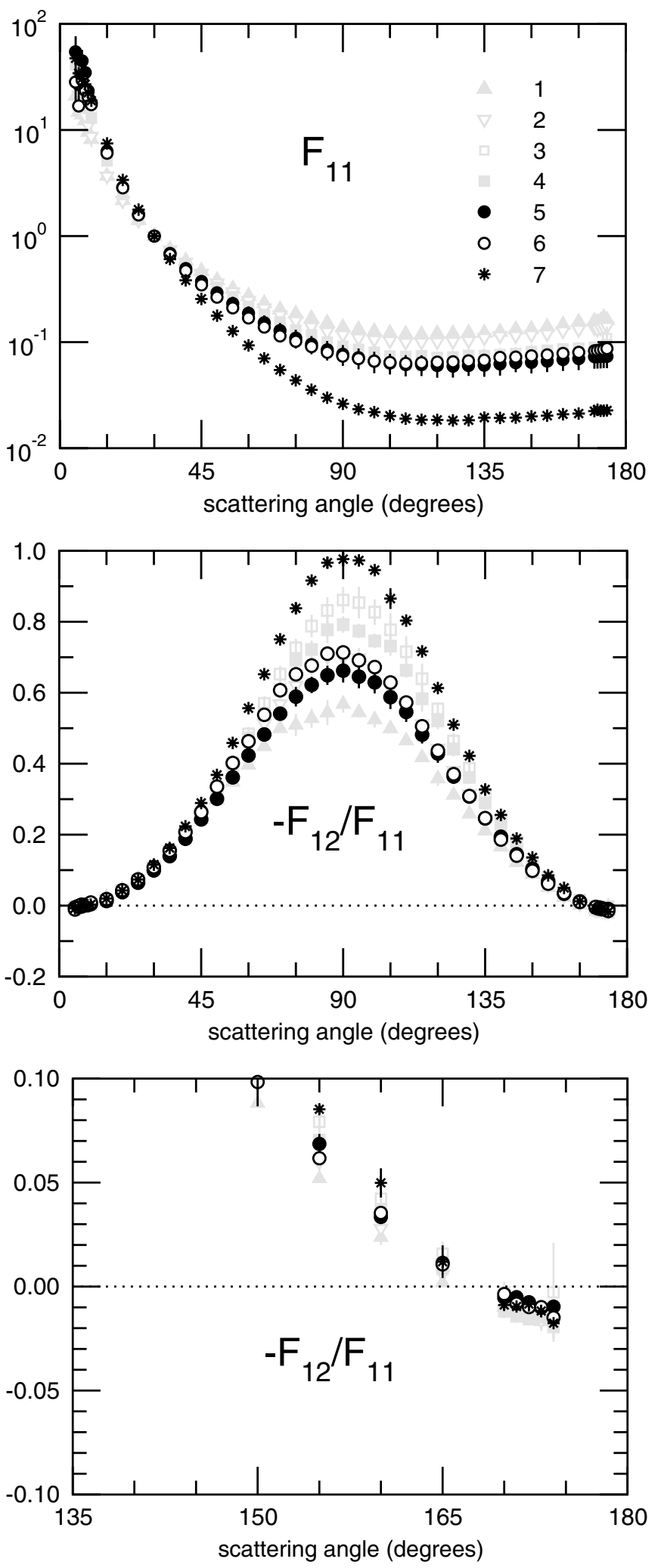

Fig. 8. Measured angular distributions of the scattering matrix elements $F_{11}(\theta)$ and $-F_{12}(\theta) / F_{11}(\theta)$ at $632.8 \mathrm{~nm}$ for a ferrosilica sample (sample 5, solid circles), a ferrosilica-magnesiosilica sample (sample 6, open circles) and an alumina sample (sample 7, stars). As a reference, the magnesiosilica results from Fig. 7 are shown in the background in grey. In the bottom panel an enlargement of the measured $-F_{12}(\theta) / F_{11}(\theta)$ at large scattering angles is shown. If no error bars are visible, errors are smaller than the symbols. The data can be found in numerical form in "The Amsterdam Light Scattering Database" (http://www . astro.uva.nl/scatter). results were found before, both experimentally, e.g. a maximum $-F_{12}(\theta) / F_{11}(\theta)$ of up to about $90 \%$ for samples of fluffy alumina and silica aggregates built of submicron grains (Hadamcik et al. 2003, 2006) and numerically e.g. for several types of aggregates built from submicron-sized spheres (West 1991; Liu $\&$ Mishchenko 2005). We can view these results from two different angles. First, we can say that the $-F_{12}(\theta) / F_{11}(\theta)$ and $F_{22}(\theta) / F_{11}(\theta)$ ratios for the fluffy aggregate analog samples approach their small particle (Rayleigh) limit (Van de Hulst 1957; Bohren \& Huffman 1983). It suggests that the sizes of the individual grains are the determining factor for these matrix elements. In general, the smaller the size of the individual grains the higher the maximum of $-F_{12}(\theta) / F_{11}(\theta)$ (cf. Hadamcik et al. 2006). For the phase functions $F_{11}(\theta)$ it seems that the dimensions of the aggregate as a whole is the dominant factor, and this function may be viewed as a first indicator of macroscopic size. This is an attractive way to interpret the measurements since it means that different matrix elements carry information about different properties of the particles.

Second, we can observe that the high steepness of the phase functions combined with the high maxima of $-F_{12}(\theta) / F_{11}(\theta)$ are reminiscent of Rayleigh-Gans behavior, i.e. the fluffy particles studied here behave like particles relatively large compared to the wavelength and with an index of refraction close to one (Van de Hulst 1957; Bohren \& Huffman 1983). This makes sense if we assume that the high fluffiness of the aggregates (see Fig. 6) causes a low effective refractive index.

Both interpretations explain why the alumina sample 7 with its extremely loose structure has the most perfect Rayleigh-Gans behavior in the polarization of the seven samples; this loose structure also causes the small grains that built the aggregates to be more "visible". Also, there probably are fewer small, dense aggregates that might act as large fused grains in the alumina sample 7 than in the condensed silicate samples 1-6 (see Sects. 3.3 and 3.4).

Recent results of Hadamcik et al. (2007) indicate that if the aggregates become very large, i.e. millimeter-sized, the degree of linear polarization may decrease again. They performed measurements of the degree of linear polarization for unpolarized incident light of magnesiosilica samples produced with the same apparatus as our samples and found maxima of around only $35-40 \%$. The difference between their results and ours is probably mainly due to the fact that they use agglomerates of aggregates with sizes up to millimeters instead of micron-sized particles.

Interestingly, for the negative branch of $-F_{12}(\theta) / F_{11}(\theta)$ at large scattering angles we see practically the same behavior for all seven samples, i.e. minima of around 1-2\% (see bottom panel Fig. 8). These minima do not seem to be strongly correlated to grain size, aggregate size, or composition. For example, if the grain size would determine the negative branch, this branch would be expected to be slightly positive for sample 7 as for Rayleigh or Rayleigh-Gans scattering. Instead aggregate fluffiness seems to be the main cause for the negative branch, since for compact irregular minerals of silicate compositions this negative branch is usually a few percent deeper (Volten et al. 2006b). Although we mostly confirm the findings of Hadamcik et al. (2006) for the maxima of $-F_{12}(\theta) / F_{11}(\theta)$ for fluffy aggregates, they do not find the same uniformity as we do for the negative branch. They measured no negative branch for pure materials, such as pure alumina or silica, and larger differences for negative branches for mixtures of materials, such as silica mixed with alumina and silica mixed with carbon. 
Table 3. Properties per sample.

\begin{tabular}{llllll}
\hline \hline sample & main components & n and color & fluffiness & modal grain sizes & aggregate/cluster sizes \\
\hline 1 & $95 \% \mathrm{MgSiO}, 5 \% \mathrm{MgO}$ & 1.7 dark brown & high & $50 \mathrm{~nm}, 120 \mathrm{~nm}$ & about $650 \mathrm{~nm}$ \\
2 & $95 \% \mathrm{MgSiO}, 5 \% \mathrm{MgO}$ & 1.7 dark brown & high & $50 \mathrm{~nm}, 120 \mathrm{~nm}$ & about $650 \mathrm{~nm}$ \\
3 & $75 \% \mathrm{MgSiO}, 25 \% \mathrm{SiO}$ & 1.6 light brown & high & $50 \mathrm{~nm}, 30-50 \mathrm{~nm}$ & about $650 \mathrm{~nm}, 150-400 \mathrm{~nm}$ \\
4 & $75 \% \mathrm{MgSiO}, 25 \% \mathrm{SiO}$ & 1.6 light brown & high & $50 \mathrm{~nm}, 30-50 \mathrm{~nm}$ & about $650 \mathrm{~nm}, 150-400 \mathrm{~nm}$ \\
5 & $\mathrm{FeSiO}$ & 1.8 black & higher & $15-60 \mathrm{~nm}$ & $<400 \mathrm{~nm}$ \\
6 & $\mathrm{MgSiO}, \mathrm{FeSiO}$ & 1.7 black & high to higher & $50 \mathrm{~nm}, 15-60 \mathrm{~nm}$ & $<650 \mathrm{~nm}$ \\
7 & $\mathrm{AlO}$ & 1.7 white & extremely high & $50 \mathrm{~nm}$ & micron-sized \\
\hline
\end{tabular}

The compositions of the fluffy aggregates and consequently their complex refractive indices probably play only a minor role in explaining the differences in scattering behavior among the seven samples. We see for instance only a small difference between the samples 5 ( $\mathrm{FeSiO}$ ) and 6 (a FeSiO-MgSiO mixture), and the light scattering properties of both samples are similar to that of the magnesiosilica aggregate samples 1-4 (cf. also Table 2). This result comes as no surprise when we look at the estimated values for the real part of the refractive index listed in Table 3 that are within the narrow range of 1.6-1.8. We do not have values for the imaginary part of the refractive index, but the color variations between the samples, from white to black, indicate that a great difference among the samples may exist in this respect. Apparently, for such small particles as described here the imaginary part of the refractive index is relatively unimportant compared to the real part (see also Muñoz et al. 2006; Hadamcik et al. 2006). This is also in agreement with the aggregate model results of Petrova et al. (2004) who find that the polarization is more sensitive to the real part of the refractive index than to its imaginary part.

The compositions of the grains in the aggregates do play a potentially important role in an indirect way by affecting aggregate size and structure. Unraveling in detail the interrelationships of aggregate size and structure and the effects due to the refractive index will not be a straightforward task. To start simple, the differences in maxima of $-F_{12}(\theta) / F_{11}(\theta)$, and minima of $F_{22}(\theta) / F_{11}(\theta)$ for the dark-brown magnesiosilica samples 1 and 2 compared to the light-brown samples 3 and 4 may be caused by differences in their grain sizes. The light-brown magnesiosilica samples could have higher maxima because they have a higher amount of relatively small $\mathrm{SiO}_{2}$ grains, whereas the darkbrown samples 1 and 2 contain relatively large $\mathrm{MgO}$ grains (see Table 3). More into detail, we also see differences in maxima of $-F_{12}(\theta) / F_{11}(\theta)$ between the chemically identical samples 1 and 2 , and - even stronger - for the samples 3 and 4 . Since the two dark-brown samples 1 and 2 are otherwise the same, just as the two light-brown samples 3 and 4, it may be that the different maxima for samples 1 and 2, respectively 3 and 4, are caused by small differences in aggregate/cluster porosities. Its extremely loose structure and consequently extremely high fluffiness also exerts its influence for the alumina sample 7, which has similar or larger grain sizes than in the magnesiosilica samples 3 and 4 and yet the alumina sample has a higher maximum in $-F_{12}(\theta) / F_{11}(\theta)$. For the iron containing samples 5 and 6 the possible explanation could be more complex. The samples 5 and 6 have similar grain sizes and higher fluffiness than the lightbrown magnesiosilica samples 3 and 4 and yet their maxima of $-F_{12}(\theta) / F_{11}(\theta)$ are lower. Here it could be that the larger real part of the refractive index of the iron containing samples causes the maxima to be lower (Muñoz et al. 2006; Hadamcik et al. 2006). This may also be an alternative or additional explanation for the lower $-F_{12}(\theta) / F_{11}(\theta)$ maxima of the dark brown samples
(1 and 2) with respect to the higher maxima of the light brown samples (3 and 4).

For the phase functions $F_{11}(\theta)$ we expected that the aggregate size would play a role because, in principle, for larger particles a greater steepness in the forward scattering peak is predicted (Van de Hulst 1957; Bohren \& Huffman 1983). We did not measure at scattering angles smaller than 5 degrees where this steepness in the peak may be best visible. For compact irregular silicate particles we observed over the measured angle range that the phase function generally becomes flatter for larger particles (e.g. Volten et al. 2001). This trend seems to fit the fluffy aggregate samples $1-6$ as well. That is, the larger magnesiosilica aggregates (samples 1 and 2) show a flatter phase function than the smaller magnesiosilica aggregates (samples 3 and 4). The iron containing samples 5 and 6 have a still smaller aggregate size and show a still higher steepness (see Table 2). Unfortunately, we have no estimate for the dimensions of the aggregates in the alumina sample.

In short, providing a thorough explanation of these new experimental results poses an important challenge for theoretical studies of the scattering behavior of fluffy aggregates; these studies are currently in progress.

\section{Conclusions}

With our results we hope to have made a valuable contribution to a field with a great dearth of experimental results. The results are presented in graphical form. In addition, the data can be found in numerical form in "The Amsterdam Light Scattering Database" (http://www . astro. uva.nl/scatter) for use in further research. Our results are for well documented cosmic dust analogs, i.e. with compositions and structures closely resembling that expected in cosmic environments, such as comets. For these fluffy aggregates we obtained for the most part highly accurate light scattering results for a fine mesh of scattering angles. A strong point of our results is also that we measured a combination of phase functions and polarization. Although phase functions can seldomly be determined for e.g. comets, since the number of particles in the coma cannot be assumed to be constant in time, it holds great potential for the interpretation of observations if both quantities are taken into account at the same time, for example through the use of model calculations (Moreno et al. 2002; Lasue \& Levasseur-Regourd 2006). Observations of the degree of linear polarization for incident unpolarized (sun) light for comets show values (Chernova et al. 1993; Rosenbush et al. 1994; Manset \& Bastien 2000; Levasseur-Regourd \& Hadamcik 2003) that are mostly intermediate between the values of the fluffy particles described here, and the compact irregular silicate particles studied by e.g. Volten et al. $(2005,2006 a, b)$ and Hadamcik et al. (2006), most noticeable, perhaps, for the negative branch. By taking the phase function into account, it becomes possible to distinguish whether this is caused by the 
presence in comets of certain types of fluffy aggregates, or compact irregular silicate particles, or perhaps by particles of an entirely different nature.

In particular, the fact that aggregate size seems to determine largely the phase functions, the grain size the maximum in the degree of linear polarization for unpolarized incident light, and the structure of the aggregates the negative branch, seems to hold great potential for the interpretation of observations of astronomical objects containing small particles. Indeed, this would make it possible to distinguish e.g. between small (nanometer-sized) compact particles, large (micron-sized) fluffy particles and large (micron-sized) compact particles, in particular when combined with e.g. thermal studies, or infrared spectroscopy. The presence of such different types of particles in astronomical environments such as circumstellar disks might have strong implications for the physical mechanisms involved in the formation of cosmic dust particles e.g. ongoing dust aging or thermal dust modification, and heliocentric transportation towards and away from the central star (Nuth et al. 2000b; Hill et al. 2001).

Acknowledgements. The authors would like to thank the reviewer Edith Hadamcik for her constructive comments that helped to improve this paper. F. J. M. Rietmeijer was supported by the National Aeronautics and Space Administration under Grants NAG5-11762 and NNG05GG10G issued through the Office of Space Science, and by RTOPS from the Cosmochemistry and Origins of Solar Systems Research Programs. The work of O. Muñoz has been partially supported by contracts AYA2004-03250 and ESP2003-00357. J. A. Nuth is grateful for the support he has received from the Cosmochemistry Program at NASA Headquarters. The transmission electron microscope analyses were performed in the Electron Microbeam Analyses Facility at UNM Department of Earth and Planetary Sciences. Drs Natasha Johnson, Yuki Kimura, Sue Hallenbeck, and Paul Whitey assisted with the production of the samples at the NASA Goddard Space Flight Center. Aurora Pun helped with producing the transmission electron microscope images at UMN. Ben Veihelmann produced the scanning electron microscope images at FOM-institute AMOLF, Amsterdam.

\section{References}

Anders, E., \& Grevesse, N. 1989, Geochim. Cosmochim. Acta, 53, 197

Begemann, B., Dorschner, J., Henning, T., et al. 1997, ApJ, 476, 199

Bohren, C. F., \& Huffman, D. R. 1983, Absorption and Scattering of Light by Small Particles (New York: John Wiley)

Bouwman, J., Meeus, G., de Koter, A., et al. 2001, A\&A, 375, 950

Brownlee, D., Tsou, P., Aléon, J., et al. 2006, Science, 314, 1711

Chernova, G. P., Kiselev, N. N., \& Jockers, K. 1993, Icarus, 103, 144

Cuzzi, J. N., Hogan, R. C., Paque, J. M., \& Dobrovolskis, A. R. 2001, ApJ, 546, 496

Deer, W. A., Howie, R. A., \& Zussman, J. 1974, An Introduction to the RockForming Minerals (Longman Group Limited, London)

Egan, W. G., \& Hilgeman, T. W. 1979, Optical Properties of Inhomogeneous Materials: Applications to Geology, Astronomy, Chemistry, and Engineering (New York: Academic)

Flynn, G. J., Keller, L. P., Feser, M., Wirick, S., \& Jacobsen, C. 2003, Geochim. Cosmochim. Acta, 67, 4791

Greenberg, J. M., \& Gustafson, B. A. S. 1981, A\&A, 93, 35

Hadamcik, E., Renard, J. B., Levasseur-Regourd, A. C., \& Worms, J. C. 2003, J. Quant. Spec. Radiat. Transf., 79-80, 679

Hadamcik, E., Renard, J. B., Levasseur-Regourd, A. C., \& Lasue, J. 2006, J. Quant. Spec. Radiat. Transf., 100, 143

Hadamcik, E., Renard, J. B., Rietmeijer, F. J. M., et al. 2007, Icarus [doi : $10.1016 / j$. icarus. 2007.03.010]

Hallenbeck, S. L., Nuth, J. A., \& Daukantas, P. L. 1998, Icarus, 131, 198

Hallenbeck, S. L., Nuth, III, J. A., \& Nelson, R. N. 2000, ApJ, 535, 247

Henning, T., Il'In, V. B., Krivova, N. A., Michel, B., \& Voshchinnikov, N. V. 1999, A\&AS, 136, 405

Hill, H. G. M., Grady, C. A., Nuth, III, J. A., Hallenbeck, S. L., \& Sitko, M. L. 2001, Proceedings of the National Academy of Science, 98, 2182

Hovenier, J. W. 2000, in Light Scattering by Non-Spherical Particles, ed. M. I Mishchenko, J. W. Hovenier, \& L. D. Travis (San Diego: Academic), 355
Hovenier, J. W., Volten, H., Muñoz, O., Van der Zande, W. J., \& Waters, L. B. F. M. 2003, J. Quant. Spec. Radiat. Transf., 79-80, 741

Konert, M., \& Vandenberghe, J. 1997, Sedimentology, 44, 523

Kozasa, T., \& Sogawa, H. 1997, Ap\&SS, 251, 165

Kozasa, T., \& Sogawa, H. 1998, Ap\&SS, 255, 437

Krikorian, E., \& Sneed, R. J. 1979, Ap\&SS, 65, 129

Lasue, J., \& Levasseur-Regourd, A. C. 2006, J. Quant. Spec. Radiat. Transf., 100,220

Levasseur-Regourd, A. C., \& Hadamcik, E. 2003, J. Quant. Spec. Radiat. Transf., 79-80, 903

Levasseur-Regourd, A. C., McBride, A. N., Hadamcik, E., \& Fulle, M. 1999, A\&A, 348, 636

Liu, L., \& Mishchenko, M. I. 2005, J. Geophys. Res., 110, 11211

Manset, N., \& Bastien, P. 2000, Icarus, 145, 203

Moreno, F., Muñoz, O., López-Moreno, J. J., Molina, A., \& Ortiz, J. L. 2002, Icarus, 156, 474

Muñoz, O., Volten, H., de Haan, J. F., Vassen, W., \& Hovenier, J. W. 2000, A\&A, 360, 777

Muñoz, O., Volten, H., Hovenier, J. W., et al. 2004, J. Geophys. Res., 109, 16201

Muñoz, O., Volten, H., Hovenier, J. W., et al. 2006, A\&A, 446, 525

Nuth, J. A., \& Donn, B. 1983, J. Geophys. Res., 88, A847

Nuth, J. A., \& Donn, B. 1984, J. Geophys. Res., 89, B657

Nuth, J. A., Hallenbeck, S. L., \& Rietmeijer, F. J. M. 2000a, J. Geophys. Res., 105,10387

Nuth, J. A., Hill, H. G. M., \& Kletetschka, G. 2000b, Nature, 406, 275

Nuth, J. A., Rietmeijer, F. J. M., Hallenbeck, S. L., Withey, P. A., \& Ferguson, F. 2000c, Thermal Emission Spectroscopy and Analysis of Dust, Disks, and Regoliths, ASP Conf. Ser., 196, 313

Nuth, III, J. A., Rietmeijer, F. J. M., \& Hill, H. G. M. 2002, Meteoritics and Planetary Science, 37, 1579

Petrova, E. V., Jockers, K., \& Kiselev, N. N. 2000, Icarus, 148, 526

Petrova, E. V., Tishkovets, V. P., \& Jockers, K. 2004, Sol. System Res., 38, 309

Rietmeijer, F. J. M. 1993, Earth Planet. Sci. Lett., 117, 609

Rietmeijer, F. J. M. 1998, Revs. Mineralogy, ed. J. J. Papike, Vol. 36 (Mineralogical Society of America, Chantilly, Virginia), 2-1-2-94

Rietmeijer, F. J. M. 2002, Chemie der Erde, 62, 1

Rietmeijer, F. J. M., \& Nuth, III, J. A. 1991, in Lunar and Planetary Science Conference, ed. G. Ryder, \& V. L. Sharpton, 591

Rietmeijer, F. J. M., \& Karner, J. M. 1999, J. Chem. Phys., 110, 4554

Rietmeijer, F. J. M., \& Nuth, III, J. A. 2004, in The New Rosetta Targets. Observations, Simulations and Instrument Performances, ed. L. Colangeli, E. Mazzotta Epifani, \& P. Palumbo (Astrophys. Space Sci. Library, Kluwer Academic Publishers), ASSL, 311, 97

Rietmeijer, F. J. M., Nuth, III, J. A., \& Karner, J. M. 1999a, ApJ, 527, 395

Rietmeijer, F. J. M., Nuth, III, J. A., \& Karner, J. M. 1999b, Phys. Chem. Chem. Phys., 1, 1511

Rietmeijer, F. J. M., Hallenbeck, S. L., Nuth, J. A., \& Karner, J. M. 2002a, Icarus, 156,269

Rietmeijer, F. J. M., Nuth, III, J. A., Karner, J. M., \& Hallenbeck, S. L. 2002b, Phys. Chem. Chem. Phys., 4, 546

Rietmeijer, F. J. M., Nuth, III, J. A., Rochette, P., et al. 2006, Amer. Mineral, 91, 1688

Rosenbush, V. K., Rosenbush, A. E., \& Dement'ev, M. S. 1994, Icarus, 108, 81

Thomas, K. L., Blanford, G. E., Keller, L. P., Klöck, W., \& McKay, D. S. 1993, Geochim. Cosmochim. Acta, 57, 1551

Thomas, K. L., Keller, L. P., \& McKay, D. S. 1996, in ASP Conf. Ser., 104, IAU Coll., 150, Physics, Chemistry, and Dynamics of Interplanetary Dust, ed. B. A. S. Gustafson, \& M. S. Hanner, 283

Van de Hulst, H. C. 1957, Light Scattering by Small Particles (New York: John Wiley)

Volten, H., Muñoz, O., Rol, E., et al. 2001, J. Geophys. Res., 106, 17375

Volten, H., Muñoz, O., Hovenier, J. W., et al. 2005, J. Quant. Spec. Radiat. Transf., 90, 191

Volten, H., Muñoz, O., Brucato, J. R., et al. 2006a, J. Quant. Spec. Radiat. Transf., 100, 429

Volten, H., Muñoz, O., Hovenier, J. W., \& Waters, L. B. F. M. 2006b, J. Quant. Spec. Radiat. Transf., 100, 437

Weast, R. C., Lide, D. R., Astle, M. J., \& Beyer, W. H., ed. 1998, 70th Edition CRC Handbook of Chemistry and Physics (Boca Raton, Florida: CRC Press In.)

West, R. A. 1991, Appl. Opt., 30, 5316

Zolensky, E., Wilson, T. L., Rietmeijer, F. J. M., \& Flynn, G. J., ed. 1994, Analysis of Interplanetary Dust Particles (Amer. Inst. Physics, New York), AIP Conf. Proc., 310

Zolensky, M. E., Zega, T. J., Yano, H., et al. 2006, Science, 314, 1735 